Journal of

Synchrotron

Radiation

ISSN 0909-0495

Editors: G. Ice, Å. Kvick and T. Ohta

\title{
Fast residual stress mapping using energy-dispersive synchrotron X-ray diffraction on station 16.3 at the SRS
}

\author{
Alexander M. Korsunsky, Steve P. Collins, R. Alexander Owen, Mark R. \\ Daymond, Saïda Achtioui and Karen E. James
}

J. Synchrotron Rad. (2002). 9, 77-81

Copyright (C) International Union of Crystallography

Author(s) of this paper may load this reprint on their own web site or institutional repository provided that this cover page is retained. Republication of this article or its storage in electronic databases other than as specified above is not permitted without prior permission in writing from the IUCr.

For further information see http://journals.iucr.org/services/authorrights.html

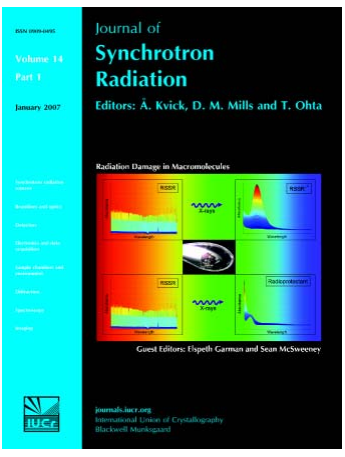

Synchrotron radiation research is rapidly expanding with many new sources of radiation being created globally. Synchrotron radiation plays a leading role in pure science and in emerging technologies. The Journal of Synchrotron Radiation provides comprehensive coverage of the entire field of synchrotron radiation research including instrumentation, theory, computing and scientific applications in areas such as biology, nanoscience and materials science. Rapid publication ensures an up-to-date information resource for scientists and engineers in the field.

Crystallography Journals Online is available from journals.iucr.org 


\section{Fast residual stress mapping using energy- dispersive synchrotron X-ray diffraction on station 16.3 at the SRS}

\author{
Alexander M. Korsunsky, ${ }^{a *}$ Steve P. Collins, ${ }^{b}$ \\ R. Alexander Owen, ${ }^{\mathrm{c}}$ Mark R. Daymond, ${ }^{\mathrm{d}}$ Saïda \\ Achtioui $^{\mathrm{a}}$ and Karen E. James ${ }^{\mathrm{a}}$
}

\author{
${ }^{a}$ Department of Engineering Science, University of Oxford, \\ Parks Road, Oxford OX1 3PJ, UK, 'b Synchrotron Radiation \\ Department, Daresbury Laboratory, Warrington WA4 4AD, UK, \\ ${ }^{c}$ Manchester Materials Science Centre, Grosvenor Street, \\ Manchester M1 7HS, UK, and 'ISIS Facility, Rutherford \\ Appleton Laboratory, Chilton, Didcot OX11 OQX, UK. \\ E-mail: alexander.korsunsky@eng.ox.ac.uk
}

Synchrotron energy-dispersive X-ray diffraction experiments on station 16.3 at the SRS for residual strain mapping are reported. A white beam with an energy-discriminating detector allows measurements to be made through $3 \mathrm{~mm} \mathrm{Al}, \mathrm{Ti}, \mathrm{Fe}$ and $\mathrm{Cu}$ alloys with acquisition times of $\sim 30 \mathrm{~s}$ per $0.3 \mathrm{~mm}^{3}$ sampling volume. The collected profiles were analysed using single-peak fitting and wholepattern Pawley refinement, and produced strain accuracy better than $10^{-4}$. This configuration is therefore highly efficient for fast strain mapping in thin components using a second-generation synchrotron source.

Keywords: residual stress; energy-dispersive X-ray diffraction.

\section{Introduction}

The use of neutron diffraction for bulk lattice strain measurement in engineering materials is well established, with many mature instruments available in Europe and around the world. In contrast, synchrotron X-ray beam instrumentation for strain scanning in engineering materials and components is less mature, and its development has been the subject of active research over the last decade. The UK teams from Oxford and Manchester have contributed to the engineering application development of high-energy monochromatic beams on station 16.3 at the SRS, Daresbury, and beamlines BM16 and ID11 at the ESRF, Grenoble (Korsunsky et al., 1998; Webster et al., 2001).

In simple terms, the higher the energy of a monochromatic beam, the lower the diffraction angle and the higher the penetration. At the medium energy of $50 \mathrm{keV}$, the one-third attenuation length varies from about $10 \mathrm{~mm}$ in $\mathrm{Al}$ down to $0.5 \mathrm{~mm}$ in $\mathrm{Fe}$ and $\mathrm{Ni}$. It follows from this observation that synchrotron X-ray beams are best suited to near-surface strain measurement, or transmission studies in light materials. However, the total photon flux on the sample, and hence the counting efficiency, can be greatly improved (potentially by a factor of $10^{4}$ or more) if a white (polychromatic) beam is used. This is due to the combined effects of using a very much larger bandwidth to collect several reflections simultaneously, and eliminating any inefficiencies associated with the monochromating optics. The set-up requires the use of a fixed solid-state detector capable of discriminating the radiation wavelength, and is known as the energy-dispersive configuration. The configuration is highly attractive to an engineer, because no displacement of detector arm is required during data collection, and focus can be firmly placed on sample translation and manipulation. Also, the detector records simultaneously a large
Table 1

Detector resolution as a function of pulse shaping time for beam energies of $5.89,22.10$ and $122 \mathrm{keV}$.

\begin{tabular}{llll}
\hline \multirow{2}{*}{ Shaping time $(\mu \mathrm{s})$} & \multicolumn{2}{l}{ Detector energy resolution $(\mathrm{eV})$} \\
\cline { 2 - 4 } & At $5.89 \mathrm{keV}$ & At $22.10 \mathrm{keV}$ & At $122 \mathrm{keV}$ \\
\hline 0.1 & 350 & 540 & 660 \\
0.5 & 290 & 420 & 530 \\
1 & 240 & 380 & 500 \\
3 & 180 & 340 & 480 \\
\hline
\end{tabular}

section of the diffraction profile containing many peaks, allowing improved insight into the average engineering strain within the sample, in the same manner as time-of-flight neutron diffraction (Korsunsky et al., 2000). It also allows the assessment of intergranular strains between the phases and grains of different orientation, which are thought to be contributing factors determining ductility and fatigue durability (Daymond et al., 1997). Studies aimed at developing successful use of energy-dispersive synchrotron X-ray diffraction for strain measurement have been reported (Pyzalla, 2000; Reimers et al., 1999). However, the strain error of the order of $4 \times 10^{-4}$ reported by Reimers et al. (1999) is too high for most engineering purposes, so serious questions remained about the ultimate strain accuracy that can be achieved by this technique.

The Bragg equation can be written in the form that relates the photon energy and lattice spacing, as follows,

$$
E d=h c /(2 \sin \theta) \text {. }
$$

In the energy-dispersive set-up, the right-hand side of this relationship is fixed, so a simple analysis shows that, for small changes of the lattice parameter, $\Delta d / d=\Delta E / E$.

The strain is calculated from the change in the lattice spacing with respect to a strain-free lattice parameter, $d_{0}$, from

$$
\varepsilon=\left(d-d_{0}\right) / d_{0} .
$$

The usual desired strain accuracy for engineering strain measurements is of the order of $\Delta d / d=10^{-4}$, or $100 \mu$ strain. This suggests that a comparable energy resolution will be required. However, modern solid-state detectors at best produce energy resolution widths of the order of $\Delta E / E=2 \times 10^{-2}$ depending on the photon energy (see Table 1), raising serious doubts about the feasibility of using this configuration for strain measurement. Recently, an exploratory experiment (SRS 37116) was carried out by the authors with the aim of investigating the possibility of overcoming this apparent limitation of the energy-dispersive set-up by optimizing the optics set-up and data-collection configuration. The principal outcomes of the exploratory study were as follows:

(i) Accurate fitting of diffraction peak shape allows the centre position to be determined to an accuracy better than 1/100th of the detector energy resolution;

(ii) Strain measurements can be performed in transmission on samples of plate and sheet form, or similar geometry;

(iii) Measurement accuracy better than $50 \mu$ strain can be achieved for both single- and multiple-peak fitting procedures;

(iv) To achieve this strain accuracy, measurements less than $10 \mathrm{~s}$ long are required;

(v) Spatial resolution of $100 \mu \mathrm{m}$ perpendicular to the beam and $1 \mathrm{~mm}$ along the beam can be achieved simultaneously.

In order to determine the limits of the technique's applicability, a range of structural alloys from $\mathrm{Al}$ to $\mathrm{Cu}$ were studied. These included unreinforced aluminium alloy (Al2124) and Al-alloy matrix composite (Al2124 and Al8090 with $20 \mathrm{vol} \% \mathrm{SiC}$ particles), Ti-6Al-4V two- 
phase aerospace alloy, ferritic steel, austenitic stainless steel, and brass.

An important observation was that the data-collection rates and the attendant strain accuracy were almost uniformly high for all of these materials. This finding does not support the conventional view that synchrotron X-ray beams are primarily useful for the analysis of near-surface stresses and/or light materials, but broadens the field of interest to include all polycrystalline structural materials. It appears that thin sections (up to $5 \mathrm{~mm}$ ) of the heavier structural materials, such as steels, and nickel and copper alloys, can also be successfully studied using the white-beam technique, opening up a new range of opportunities for applications in the automotive, power generation and other industries.

\section{Experimental}

The set-up of the instrument used for residual strain scanning using the white beam is illustrated in Fig. 1, which shows the view parallel to the incident beam. Small test samples (e.g. a bent $6 \times 3 \times 100 \mathrm{~mm}$ Ti-6-4 bar shown) were mounted on the assembly of translation and rotation tables and scanned across and/or along the beam. The diffracted signal was collected using a liquid- $\mathrm{N}_{2}$-cooled solid-state germanium detector. The sampling volume depth was defined through two pairs of crossed slits, located near the sample (front) and the detector (back). The detector position was fixed to form a small angle with the incident beam, in most cases in the range $10-15^{\circ}$. The choice of the angle was dictated by the crystal structure of the sample and the energy spectrum of the incident beam.

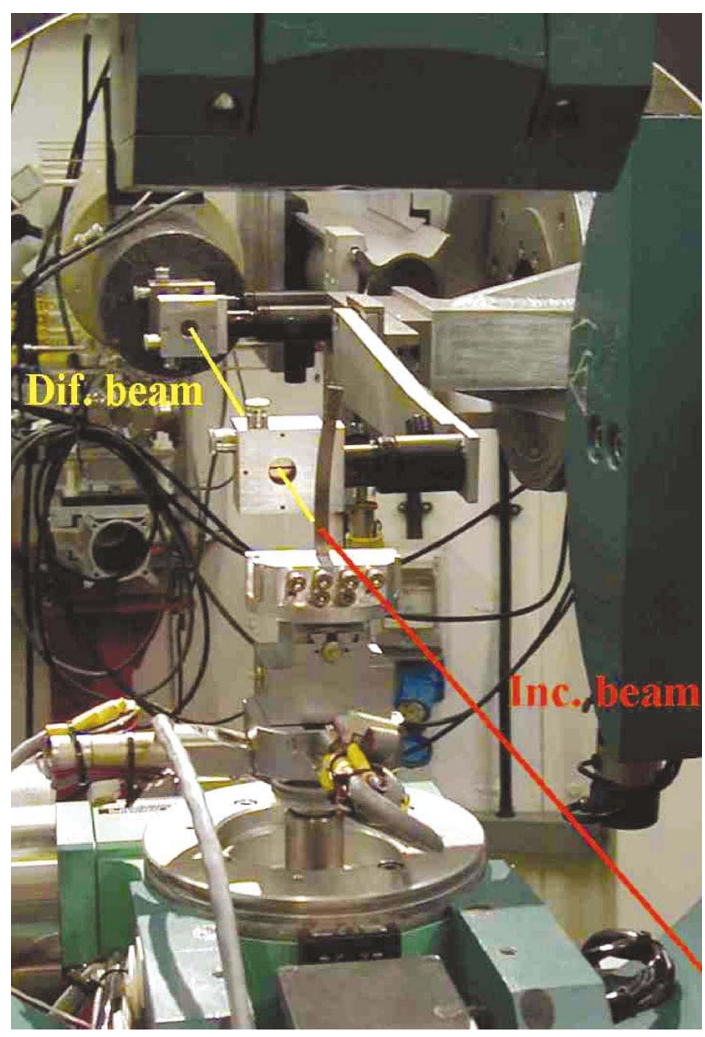

Figure 1

A view of the experimental set-up along the incident beam. Residual strain scanning was performed by translating the bent Ti-6-4 bar horizontally across the beam, and collecting the diffracted signal using a liquid- $\mathrm{N}_{2}$-cooled solidstate detector. The sampling volume depth was defined through two pairs of crossed slits, located near the sample (front) and the detector (back).
Prior to the experiment, the detector energy resolution was characterized as a function of the pulse shaping time, as shown in Table 1. A shaping time of $1 \mu$ s was found to give an adequate compromise between counting efficiency and pulse height accuracy. The energy spectrum of the diffracted beam was collected using a multichannel analyzer configured to produce 2048 output channels, and stored in a data file. The energy calibration of the detector was performed using an NBS Si 640 reference powder, and was found to be linear. The collected spectra were treated to obtain the diffraction patterns in terms of the number of counts versus photon energy or lattice spacing.

The shape of all peaks was very well approximated by the Gaussian peak function, allowing fast and reliable fitting to be performed. The data were also exported into GSAS (Larson \& Von Dreele, 1994) for a so-called Pawley refinement. This differs from a true Rietveld refinement (Rietveld, 1969) in that the peak heights are allowed to vary freely. This limits the use of the data for texture or phase volume measurements, but is useful for strain measurements since it avoids the requirement of a description of the wavelength and materialdependent absorption, which would be required for full analysis of such white-beam X-ray data. Best-fit average values of lattice parameters were obtained, and strain values calculated.

\section{Results and discussion}

In the present report we focus attention on the capabilities of the experimental set-up developed here, and the instrument's performance in the task of residual strain scanning, rather than on the deformation modelling or microstructural aspects of the engineering materials studied. Fig. 2 shows the strain profiles recorded from a plastically bent bar, following unloading, of Al2124 alloy, produced by the powder metallurgy route. Typical strain errors on a single-peak fit are below $100 \mu$ strain. The strain difference between reflections signifies the presence of intergranular stresses (Korsunsky et al., 2000; Daymond et al., 1997). Note also the excellent agreement observed between the 111 and 222 peak strains. The bold solid line shows the average residual strain profile computed using all peak data weighted by the multiplicity of the corresponding crystal direction. The use of information available from multiple peaks leads to a much more reliable estimate of the average macroscopic, or engineering, residual strain (Daymond et al., 1997).

In a two-phase material, such as a powder-reinforced metal matrix composite, diffraction peaks belonging to each of the phases can be studied separately. Alternatively, the entire diffraction pattern can be predicted using a crystallographic model, and optimized to achieve the best agreement. An example of such a fit for Al8090 reinforced with $20 \mathrm{vol} \% \mathrm{SiC}$ powder is shown in Fig. 3. The whole-pattern fitting procedure was also used in order to determine accurately the $a$ and $c$ lattice parameters of the dominant hexagonal $\alpha$-phase in Ti-6Al-4V alloy.

Fig. 4 shows the plots of residual strain variation across the Ti-6Al$4 \mathrm{~V}$ bent bar, following unloading, calculated on the basis of the $a$ and $c$ lattice parameters separately. The typical peak-fitting accuracy was better than $100 \mu$ strain, so that the error bars are comparable with the marker size. Remarkably close agreement is observed between $a$ and $c$ strains on the side that has experienced plastic compression, and has been left in residual tension. However, it is interesting to note that the situation is drastically different on the tensile side of the bar. Here the residual strain according to the $a$ parameter value is highly tensile, while that according to the $c$ parameter is very close to zero. This phenomenon is also observed if individual peak strains are calculated, and is likely to be indicative of the severe asymmetry of 


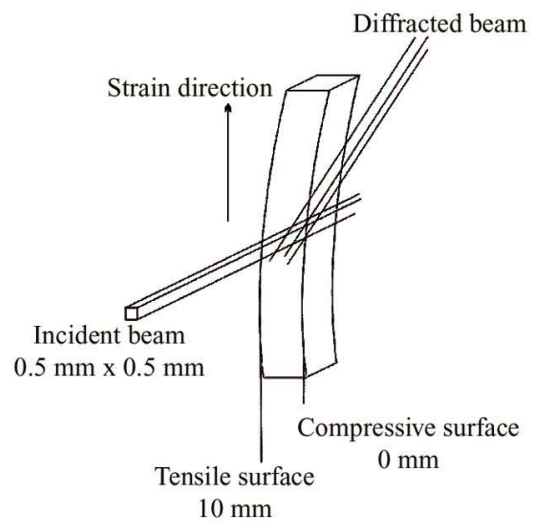

(a)

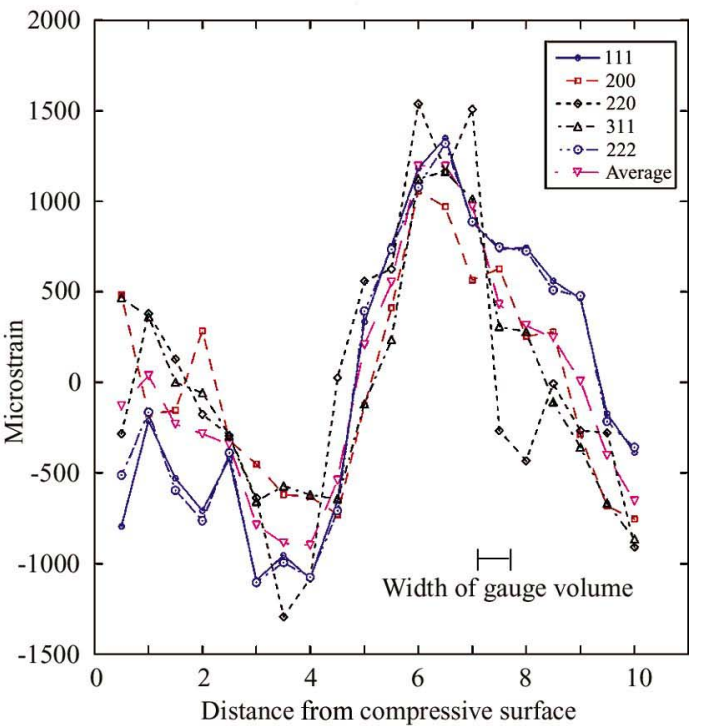

(b)

Figure 2

(a) Schematic diagram of the experimental procedure adopted, and (b) the bent bar profile of residual strain calculated using different peaks, and hence crystallographic orientations, in an Al2124 alloy. Note the strain difference between reflections, signifying the presence of intergranular stresses, and also the nature of agreement between the 111 and 222 peak strain. The bold solid line shows the average residual strain profile computed using all peak data weighted with the multiplicity of the corresponding crystal direction.

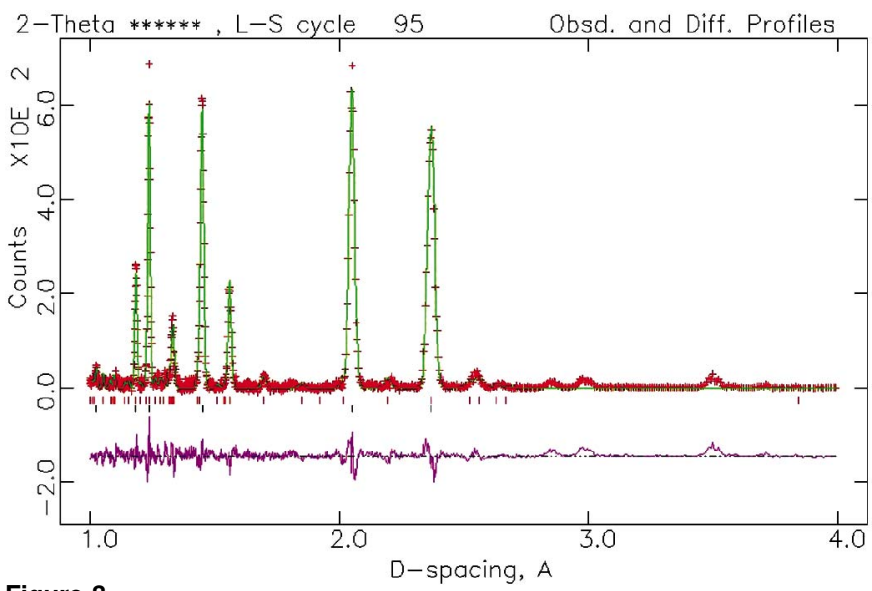

Figure 3

A GSAS Pawley two-phase fit (line) to the diffraction data (crosses) obtained from the $\mathrm{Al} / \mathrm{SiC}$ composite. The marks below indicate the predicted positions of diffraction peaks, and the bottom line is the difference between fit and data. yielding and twinning in this material with respect to the sign of loading. Data of this kind are extremely valuable for the purpose of developing and validating deformation models of polycrystals of major structural materials.

In the set-up described above, the translation assembly holding the sample was mounted on the diffractometer $\chi$-circle, allowing a $90^{\circ}$ rotation to be applied. Elasticity analysis of the stress state in thin plates, as well as direct diffraction measurements of the threedimensional strain states, confirm that the generalized plane stress assumption can be used with good justification, except when local concentrators such as cracks, notches or boreholes are present. Two mutually perpendicular components of elastic strain, $\varepsilon_{x x}$ and $\varepsilon_{y y}$, were measured. Under the conditions of generalized plane stress (assuming that out-of-plane stresses may be neglected), the corresponding stresses can be found from Hooke's law,

$$
\begin{aligned}
\sigma_{x x} & =\frac{E}{\left(1-v^{2}\right)}\left[\varepsilon_{x x}+v \varepsilon_{y y}\right], \\
\sigma_{y y} & =\frac{E}{\left(1-v^{2}\right)}\left[\varepsilon_{y y}+v \varepsilon_{x x}\right] .
\end{aligned}
$$

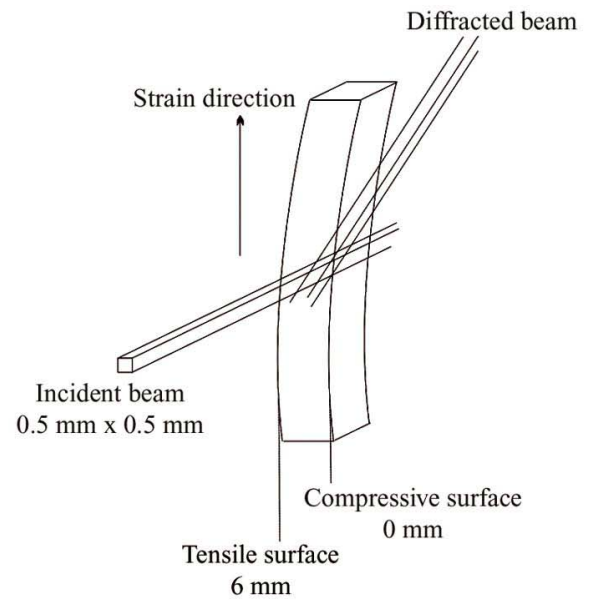

(a)

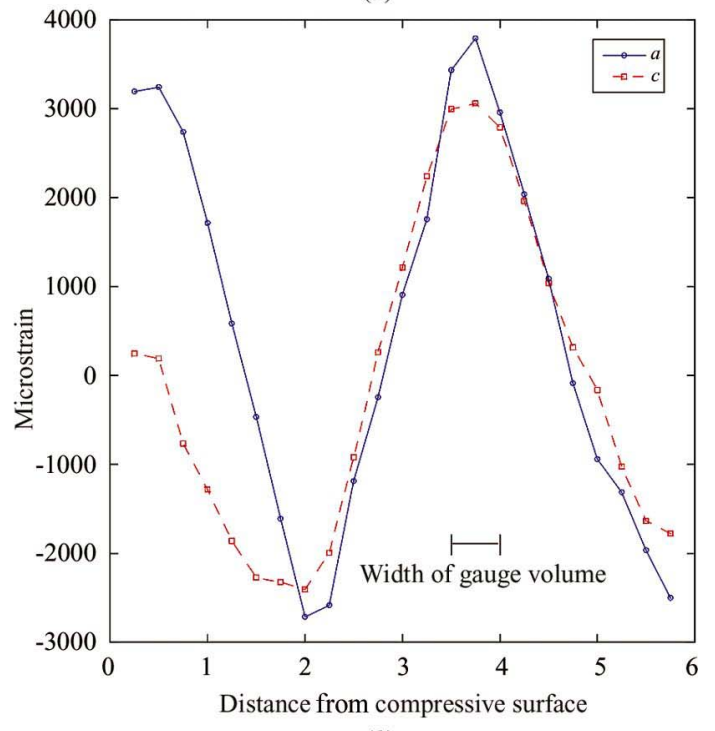

(b)

Figure 4

(a) Schematic diagram of the experimental procedure adopted, and (b) variation of the strain across a bent bar of Ti-6-4, calculated on the basis of $a$ and $c$ lattice parameters obtained from a full-pattern fit. 
Furthermore, the method allows the complete residual stress state in thin structural members to be reconstructed from two measurements of in-plane elastic strains, provided the two mutually perpendicular directions are principal, or that at least three components of strain are measured at each point (Korsunsky et al., 2002). The configuration described in the present article can therefore be used efficiently to measure engineering residual stress states in plates and shells.

However, our study demonstrates that the method is even more powerful than that. Stronger collimation of the diffracted beam allows good spatial resolution $(<1 \mathrm{~mm})$ to be achieved in the direction parallel to the beam (e.g. Fig. $5 a$ ). We used the measurement configuration described here to study the strain gradient between the sample surface and the bulk.

It is a well known fact that deformation experienced by the elements of a solid lying close to the surface is significantly different from that of the deep-lying elements within the bulk. In order to describe these conditions within the framework of two-dimensional continuum mechanics, the concepts of plane stress and plane strain are usually introduced. Material surfaces are often free from applied tractions, so that only in-plane stress components must be considered.

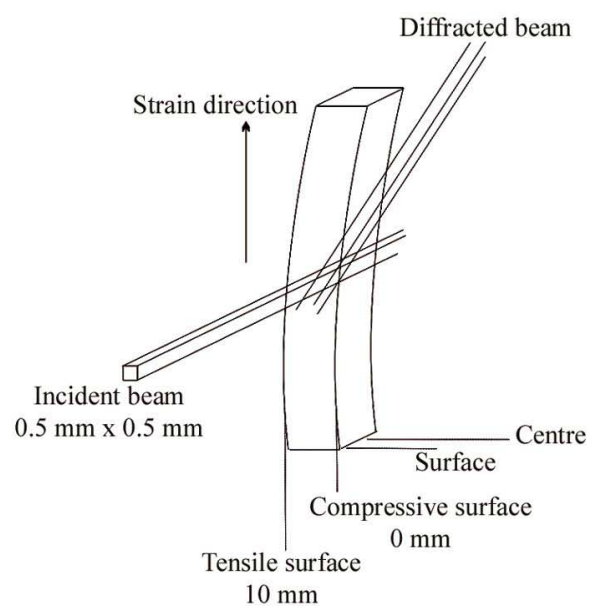

(a)

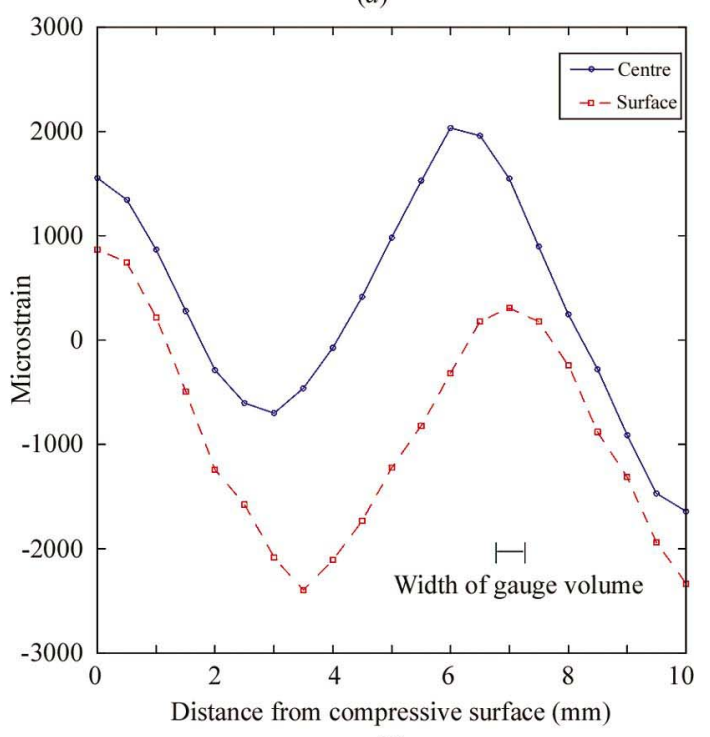

(b)

Figure 5

(a) Schematic of the experimental procedure adopted, and (b) Al strain profiles recorded at the surface and in the centre plane of an $\mathrm{Al} / \mathrm{SiC}$ bent bar.
This leads to reduced constraint and lower effective elastic stiffness of the material. As far as the plastic flow conditions are concerned, the stress component normal to the surface is often the lowest principal stress, so that plastic shear occurs on planes inclined at $45^{\circ}$ to the surface. In contrast, the constraint experienced by the bulk can be approximated by assuming that no strain can develop along the direction perpendicular to the principal loading plane, so that only inplane strains are allowed. In this case, plastic shear is also constrained to occur within the same plane.

The difference in the deformation constraint is also important in the process of residual stress generation, for example, during quenching of the sample from heat-treatment temperature. Not only thermal gradients give rise to a mismatch in the 'stress-free' dimensions of different layers, but their propensity to relieve the mismatch by plastic deformation is different.

The consequences of the difference in the level of constraint experienced by the surface and bulk elements are clearly observed under the conditions of significant plastic deformation. However, the effect is much more difficult to capture experimentally in the essentially elastoplastic regime, when the elastic and plastic strain contri-

Compressive surface

Tensile surface

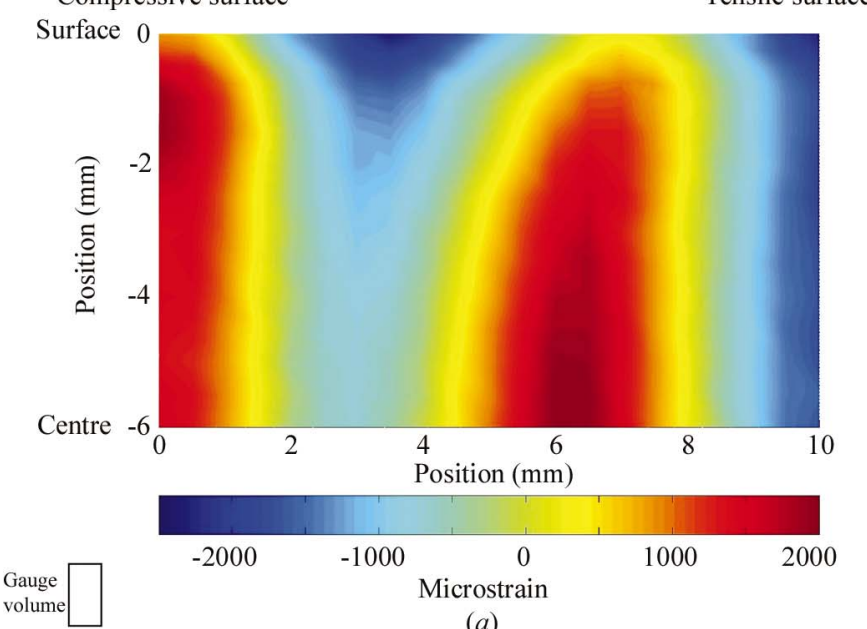

Compressive surface

(a)

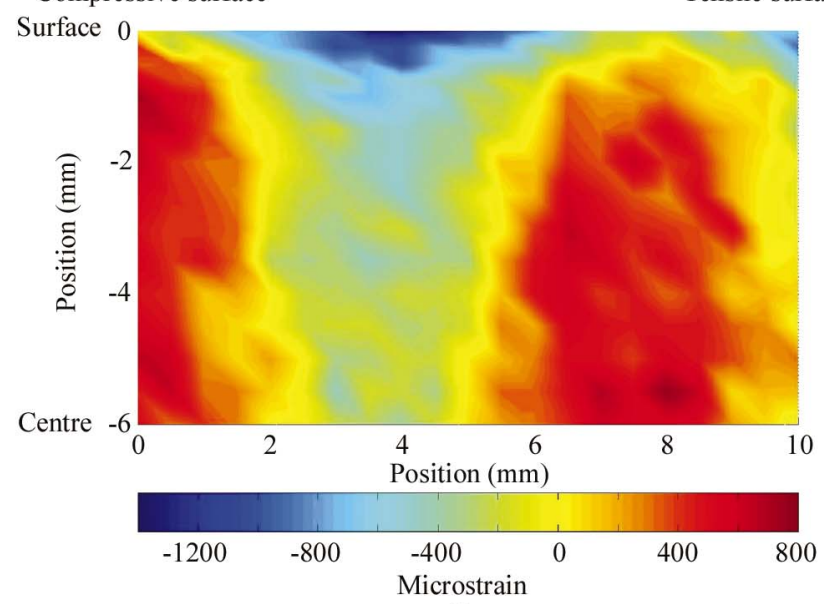

(b)

Figure 6

Strain maps showing residual bending strain transition from the surface to the centre of a bent Al-SiC MMC bar, for the matrix phase (Al8090 alloy) and the reinforcement ( $\mathrm{SiC}$ particulate). The top of the map $(0 \mathrm{~mm}$ on the vertical axis) is at the bar side face, while the bottom of the map $(-6 \mathrm{~mm})$ corresponds to the centre plane parallel to the side face. 
butions are much closer in magnitude. The majority of experimental techniques measure strain at the sample surface, and try to make inferences about deformation throughout the piece.

In order to highlight the capability of the synchrotron strain mapping technique and to capture the nature of the plane-stress to plane-strain transition, a $14 \mathrm{~mm}$-thick sample of particulate SiCreinforced $\mathrm{Al} \mathrm{MMC}$ was solution heat treated, quenched in cold water, and subjected to bending. In this way a complex residual stress state was generated within the cross section of the bar, consisting of the zigzag-shaped plastic bending residual stress profile superimposed over the quench stress distribution. The procedure guarantees a significant difference between the surface and bulk residual stress states due to either process. It is also clear that the variation of the residual stress state from the bar side surface and towards the bulk must develop due to the difference in the constraint, while a more uniform bending stress profile should be established over a large region within the bulk of the bar.

Measurements of the bending strain following unloading were performed in transmission, with the sampling volume having the dimension of approximately $1 \mathrm{~mm}$ along the beam. This allowed the strain profiles near the sample surface and in the bulk to be compared. Fig. 5 shows these profiles for the aluminium alloy matrix. The typical strain measurement error was about $50 \mu$ strain, corresponding to the size of the markers on the plots. A clear difference between the two profiles is observed, suggesting that the transition can be characterized in detail.

To provide further illustration of the method's capability, twodimensional maps of strain within each of the phases across the sample section are shown in Figs. 6(a) (matrix) and 6(b) (reinforcement). The pattern for the minority $\mathrm{SiC}$ phase (17 vol\%) is more subject to measurement uncertainty and noise, but is nevertheless sufficiently clear to demonstrate the main features of the strain variation. The bending axis is vertical, with the right-hand side of the mapped region subjected to tension and the left-hand side to compression. After load removal, the sense of residual strain that arose became opposite to that applied during loading. In the maps, the top line parallel to the horizontal axis (labelled zero) denotes the bar side surface, while the horizontal line at the bottom of the map $(-6 \mathrm{~mm})$ corresponds to the centre plane of the bar. A rapid and significant variation of the residual bending strain profile is observed in the vicinity of the sample surface. At a depth of about $2 \mathrm{~mm}$ the strain profile becomes largely stabilized and corresponds to that within the bulk of the sample, although a small variation continues to be observed.

The results provide compelling evidence of the technique's capability to resolve the surface-bulk stress state transition. The availability of such an experimental technique is of great use for numerical model validation and fatigue lifing analysis.

\section{Conclusions}

The paper describes the experimental configuration for synchrotron energy-dispersive X-ray diffraction strain measurement on station 16.3 at the SRS, Daresbury. The results presented demonstrate the significant potential benefit of using white beam in combination with an energy-dispersive detector to record large sections of powder diffraction patterns from structural engineering materials, allowing accurate and fast determination of the residual stress state.

The authors express their gratitude to Professor P. J. Withers for his advice and support for this project.

\section{References}

Daymond, M. R., Bourke, M. A. M., Von Dreele, R. B., Clausen, B. \& Lorentzen, T. (1997). J. Appl. Phys. 82, 1554-1562.

Korsunsky, A. M., Daymond, M. R. \& Wells, K. E. (2000). Mater. Sci. Forum, 347/349, 492-497.

Korsunsky, A. M., James, K. E., Aylott, C. \& Shaw, B. A. (2002). J. Strain Anal. Eng. Des. Submitted.

Korsunsky, A. M., Wells, K. E. \& Withers, P. J. (1998). Scr. Mater. 39, 17051712.

Larson, A. C. \& Von Dreele, R. B. (1994). GSAS. Los Alamos National Laboratory, New Mexico, USA.

Pyzalla, A. (2000). J. Nondestr. Eval. 19, 21-31.

Reimers, W., Pyzalla, A., Broda, M., Brusch, G., Dantz, D., Schmackers, T. \& Liss, K. (1999). J. Mater. Sci. Lett. 18, 581-583.

Rietveld, H. M. (1969). J. Appl. Cryst. 2, 65-71.

Webster, P. J., Oosterkamp, L. D., Browne, P. A., Hughes, D. J., Kang, W. P., Withers, P. J. \& Vaughan, G. B. M. (2001). J. Strain Anal. Eng. Des. 36, 61-70. 